\title{
In-Plane Optical and Electrical Anisotropy of 2D
}

\section{Black-Arsenic}

Mianzeng Zhong ${ }^{1}$, Haotong Meng ${ }^{1}$, Sijie Liu ${ }^{1}$, Huai Yang' ${ }^{2}$, Wanfu Shen ${ }^{3}$, Chunguang

$\mathrm{Hu}^{3}$, Juehan Yang ${ }^{2}$, Zhihui Ren², Bo Li ${ }^{4}$ Yunyan $\mathrm{Liu}^{5}$, Jun He${ }^{1 *}$, Qinglin $\mathrm{Xia}^{1 *}$, Jingbo

$\mathrm{Li}^{6}$, and Zhongming $\mathrm{Wei}^{2 *}$

${ }^{1}$ Hunan Key Laboratory of Super-microstructure and Ultrafast Process, School of

Physics and Electronics, Central South University, Changsha 410083, Hunan, China

${ }^{2}$ State Key Laboratory of Superlattices and Microstructures, Institute of Semiconductors,

Chinese Academy of Sciences \& Center of Materials Science and Optoelectronics

Engineering, University of Chinese Academy of Sciences, Beijing 100083, China

${ }^{3}$ State Key Laboratory of Precision Measuring Technology and Instruments, Tianjin

University, Tianjin 300072, China

${ }^{4}$ Department of Applied Physics, School of Physics and Electronics, Hunan University,

Changsha 410082, Hunan, China 
${ }^{5}$ School of Physics and Optoelectronic Engineering, Shandong University of

Technology, Zibo 255049, Shandong, China

${ }^{6}$ Institute of Semiconductors, South China Normal University, Guangzhou 510631,

China
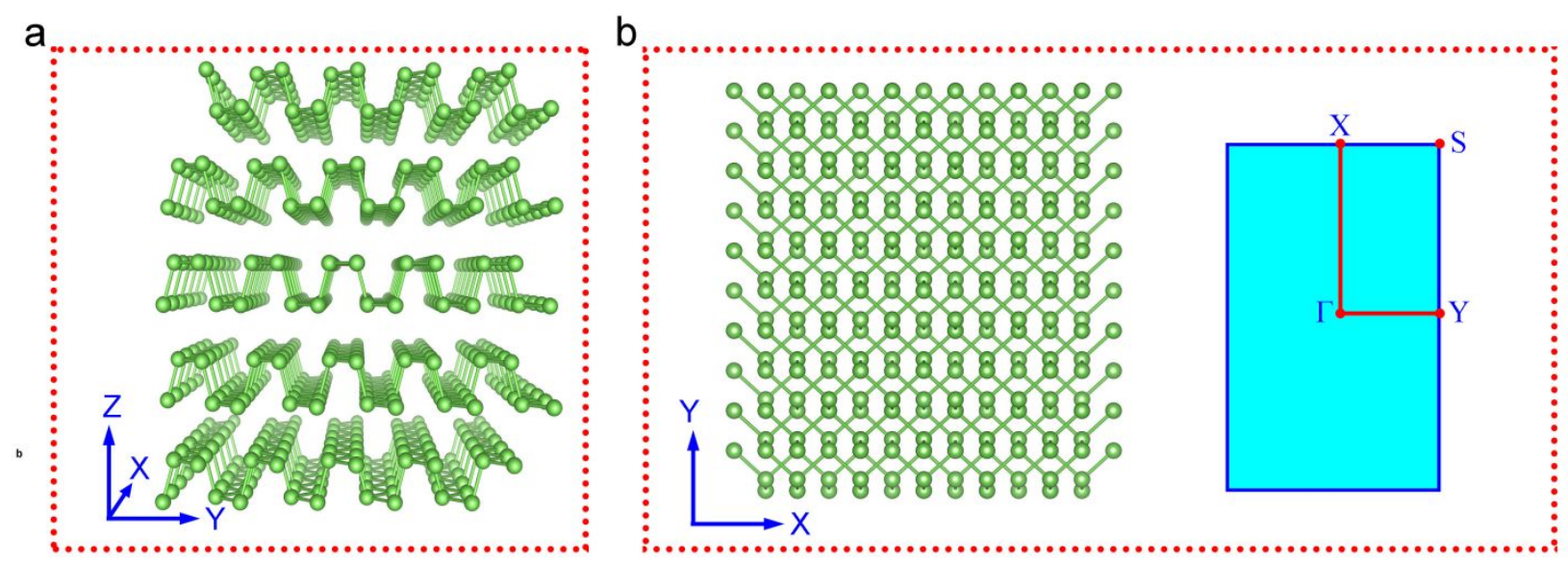

Figure S1. Crystal structures of orthorhombic b-As from (a) side view and (b) top view, showing its highly anisotropic nature. 


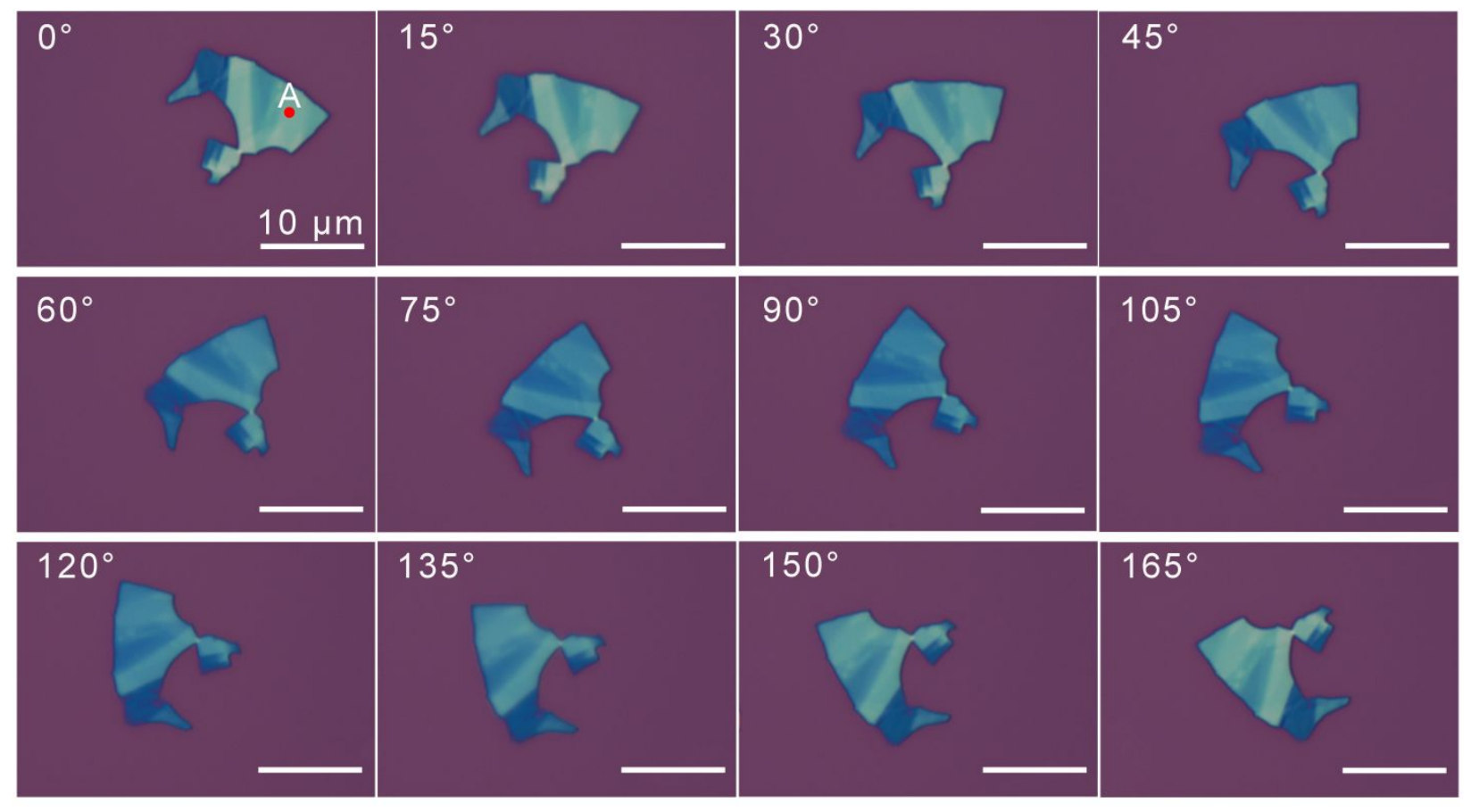

Figure S2. Polarization-resolved optical images of b-As flake.

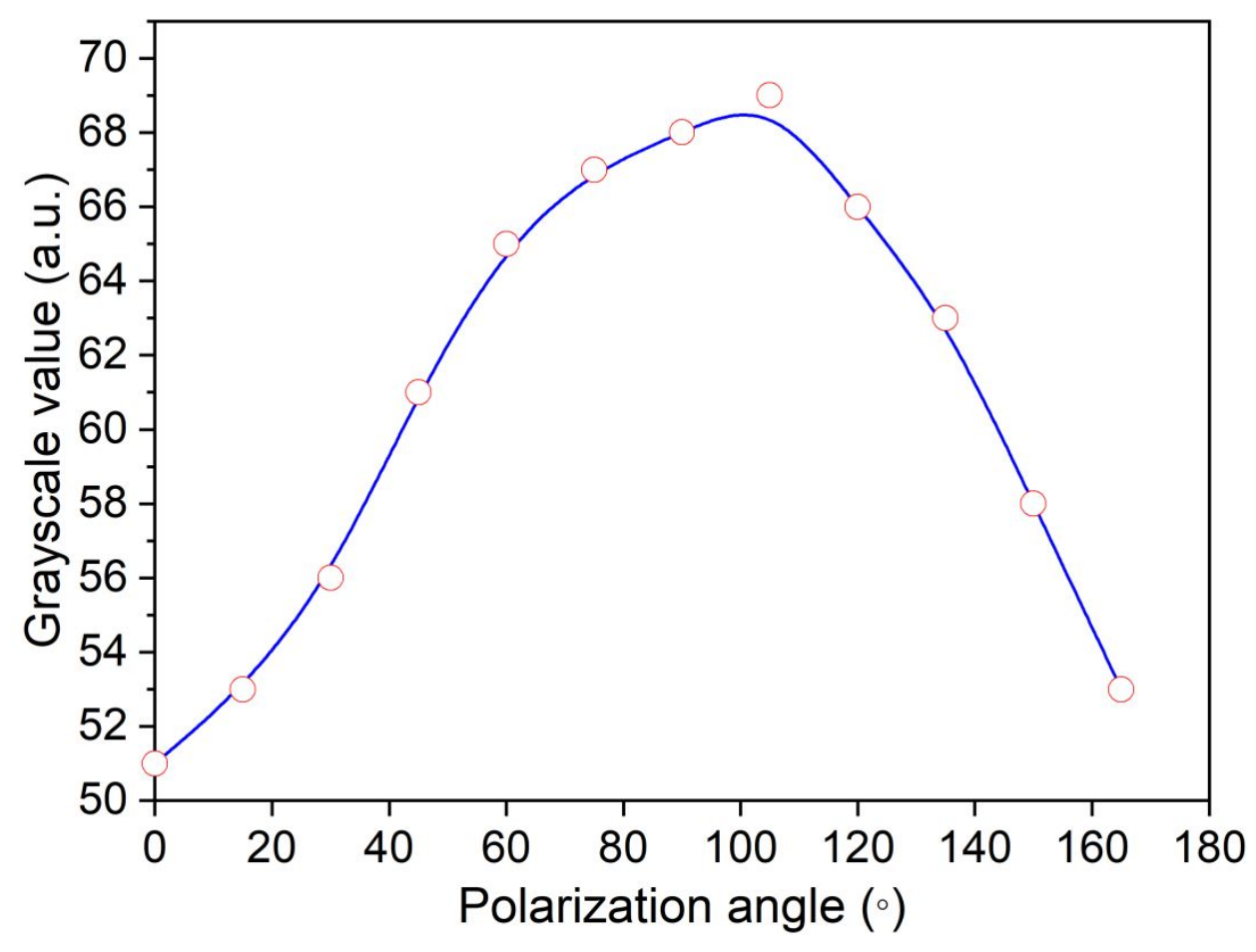


Figure S3. Grayscale values obtained from the A point in Figure S1 under different polarization angles as a function of the polarization angle.
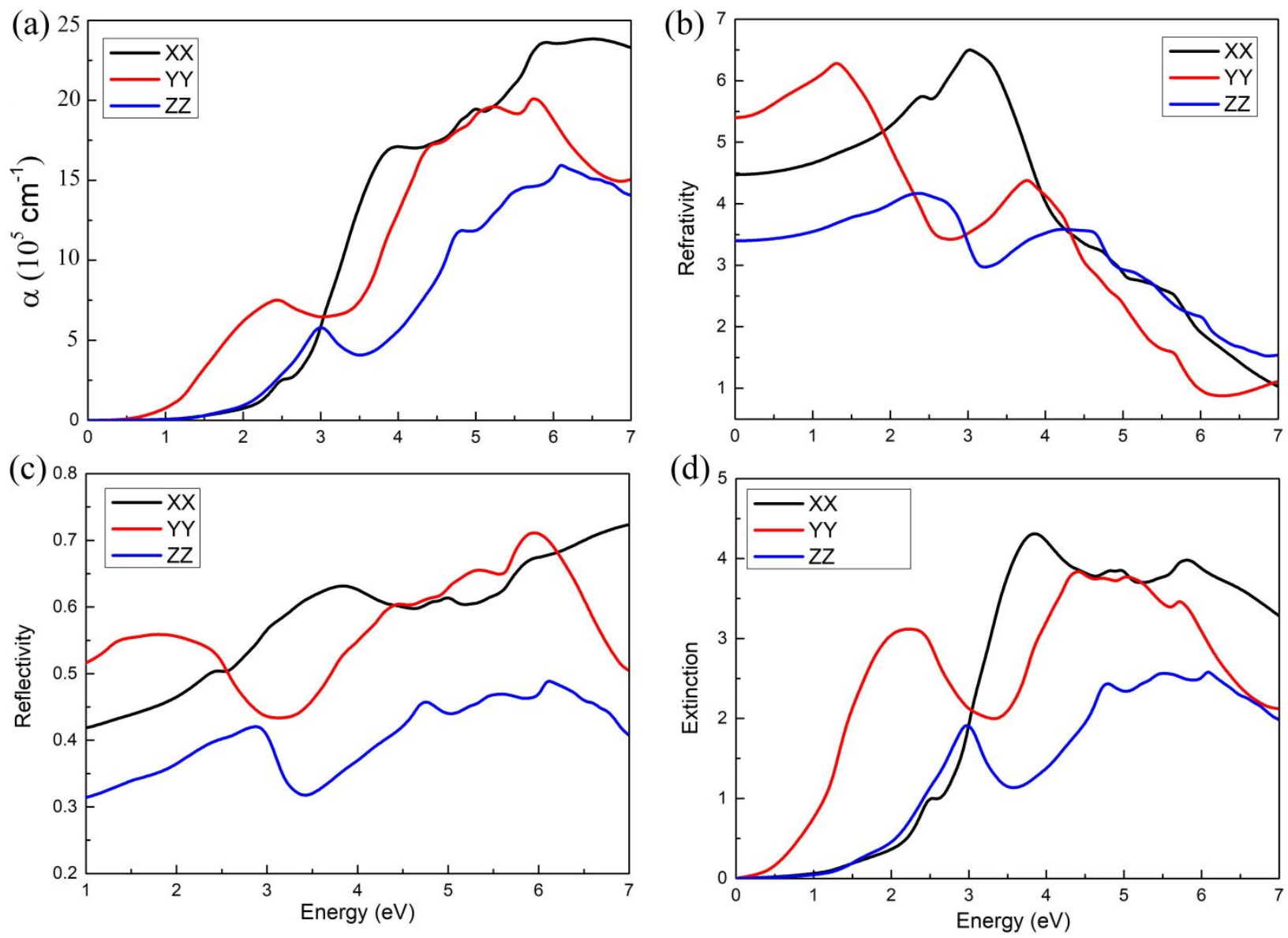

Figure S4. The absorption(a), reflectivity(b), refractive index (c), and extinction (d) for bAs considering $X X, Y Y$ and $Z Z$ directions. 

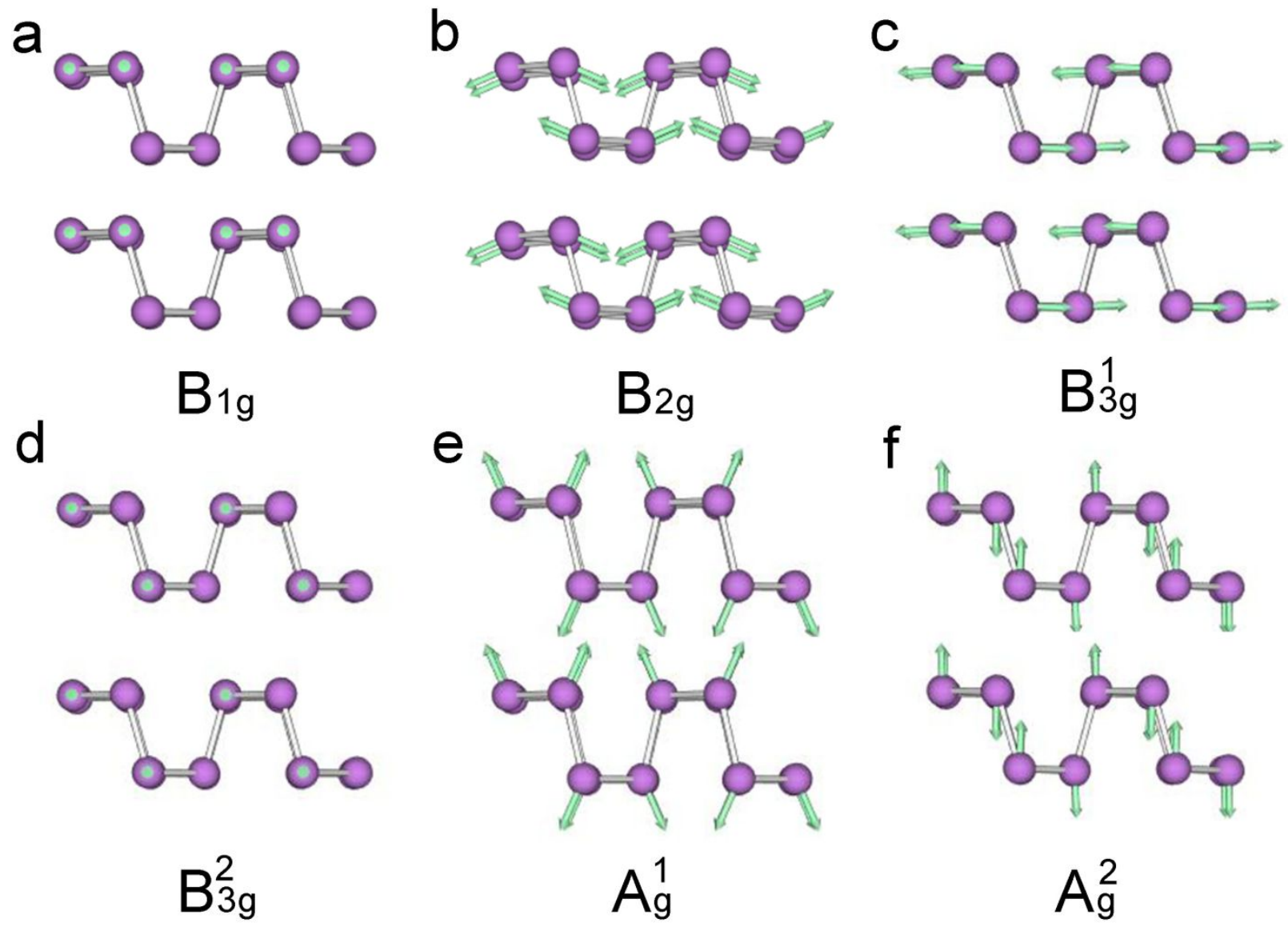

Figure S5. Schematic diagrams for six Raman-active modes in b-As. Green arrows indicate atomic displacements. 


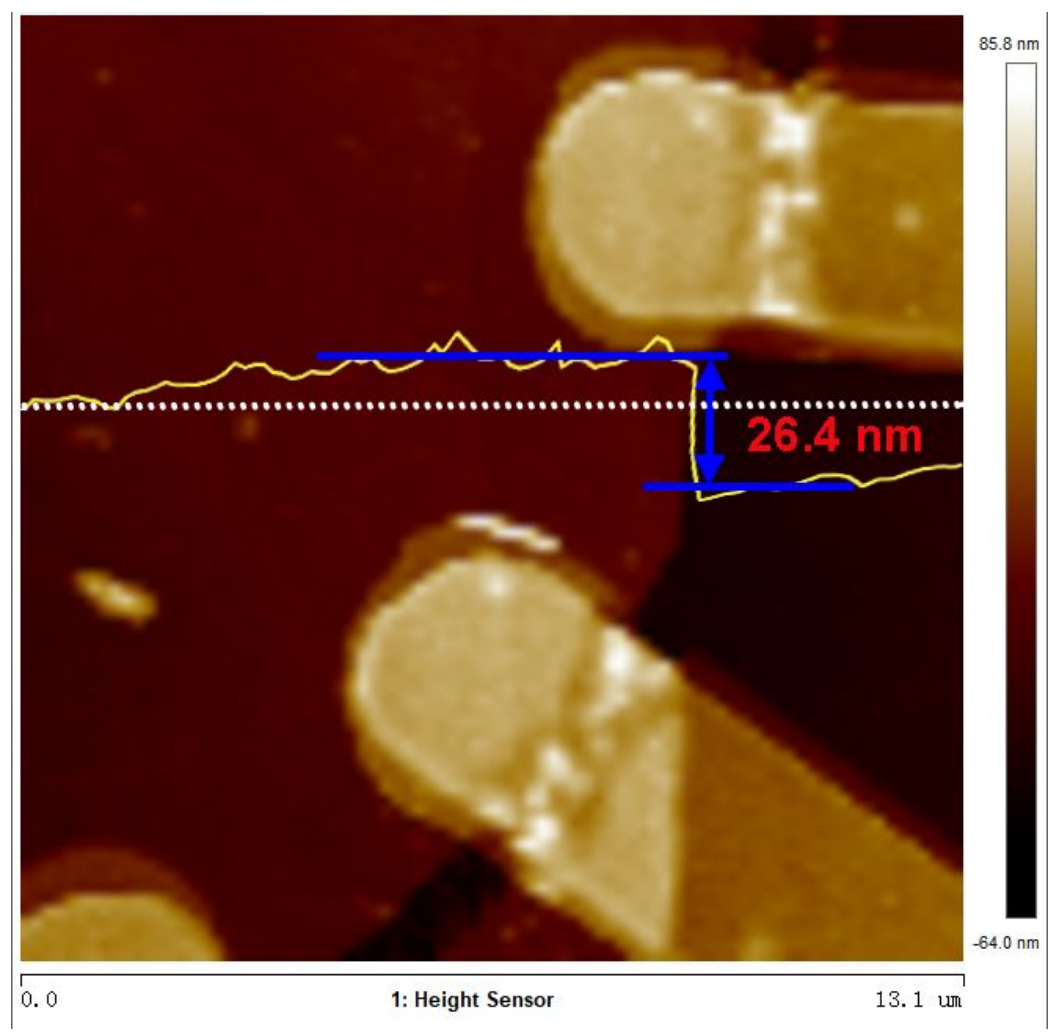

Figure S6. Atomic force microscopy (AFM) image of this typical b-As FET, the thickness of the b-As crystal is about $26.4 \mathrm{~nm}$. 


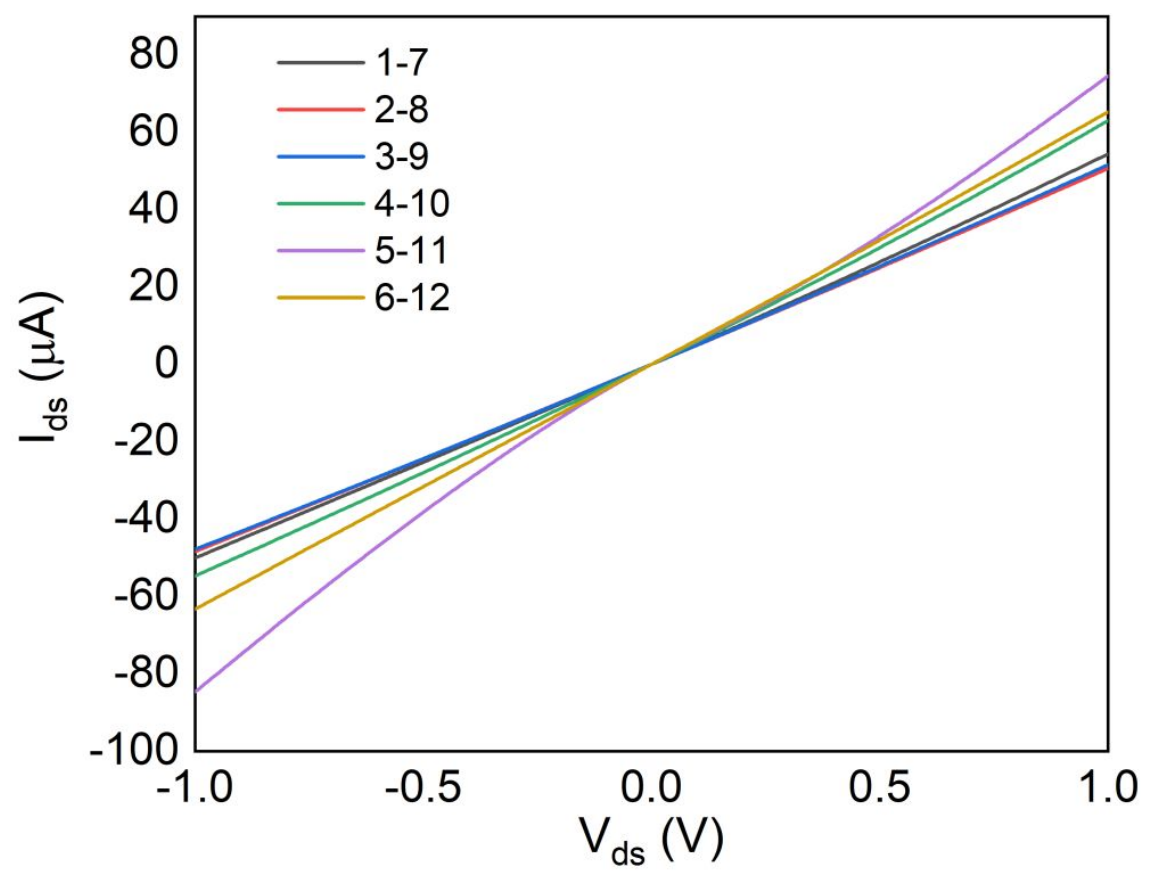

Figure S7. Room-temperature output characteristics $\left(I_{d s}-V_{d s}\right)$ of this FET with the different directions (different symmetrical source-drain electrodes), the gate voltage is OV.

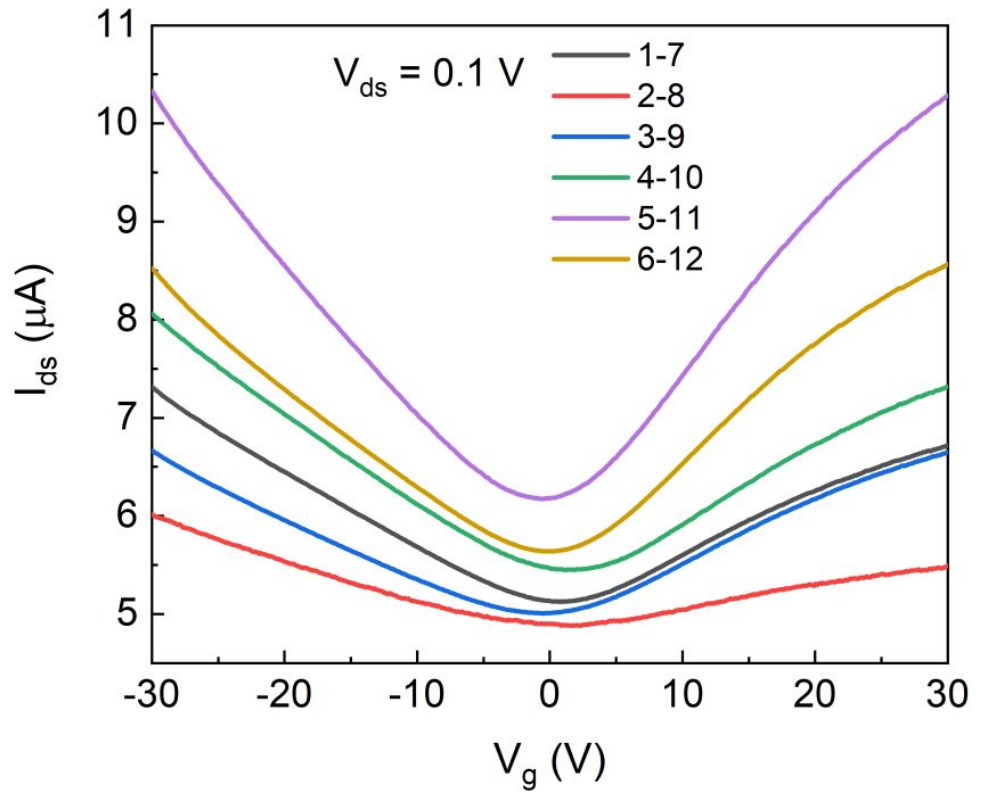


Figure S8. Room-temperature transfer characteristics $\left(I_{d s}-V_{g}\right)$ of this FET with the different directions (different symmetrical source-drain electrodes), the drain-source voltage is $1 \mathrm{~V}$.

\section{Note S1}

For b-As, the Raman tensors of the phonons with $A_{g}$, and $B_{2 g}$ symmetry are

$$
\overleftrightarrow{R}_{A_{g}}=\left(\begin{array}{lll}
a & 0 & 0 \\
0 & b & 0 \\
0 & 0 & c
\end{array}\right)=\left(\begin{array}{ccc}
|a| e^{i \emptyset_{a}} & 0 & 0 \\
0 & |b| e^{i \emptyset_{b}} & 0 \\
0 & 0 & |c| e^{i \phi_{c}}
\end{array}\right)
$$

and

$$
\overleftrightarrow{R}_{B_{2 g}}=\left(\begin{array}{ccc}
a & 0 & f \\
0 & 0 & 0 \\
f & 0 & 0
\end{array}\right)=\left(\begin{array}{ccc}
0 & 0 & |f| e^{i \emptyset_{f}} \\
0 & 0 & 0 \\
|f| e^{i \emptyset_{f}} & 0 & 0
\end{array}\right)
$$

where $|a|, \emptyset_{a},|b|, \emptyset_{b},|c|, \emptyset_{c},|f|$, and $\emptyset_{f}$ are the magnitude and complex phase of the independent components of these two tensors, respectively. In our experimental configuration, the incident laser beam along the $y$ direction (perpendicular to the layer plane of our arsenic sample). So, the polarization vector of the incident beam $\left(\hat{e}_{i}\right)$ can be expressed as $\hat{e}_{i}=(\sin \theta 0 \cos \theta)(\theta$ is the sample rotation angle $)$, and the polarization 
vector of the scattered light $\left(\hat{e}_{S}\right)$ can be expressed as $\hat{e}_{s}=(\sin \theta 0 \cos \theta)$ and $\hat{e}_{s}=(\cos \theta$ $0-\sin \theta$ ) for the parallel and cross-polarization configurations, respectively. The Raman scattering intensity, which is related to the Raman tensor and the polarization vectors of incident laser beam and scattered light, is expressed as $S \propto\left|\hat{e}_{i} \cdot \overleftrightarrow{R} \cdot \hat{e}_{S}\right|^{2}$. As a consequence, the Raman intensities of $A_{1 g}$ and $B_{2 g}$ modes under parallel and crosspolarization configurations are written as following

$$
\begin{aligned}
& S_{A_{g}}^{\prime l}=\left(|a| \sin ^{2} \theta+|c| \cos \emptyset_{c a} \cos ^{2} \theta\right)^{2}+|c|^{2} \sin ^{2} \emptyset_{c a} \cos ^{4} \theta \\
& S_{A_{g}}^{\perp}=\left[\left(|a|-|c| \cos \emptyset_{c a}\right)^{2}+|c|^{2} \sin ^{2} \emptyset_{c a}\right] \cos ^{2} \theta \sin ^{2} \theta \\
& S_{B_{2 g}}^{\prime}=|f|^{2} \sin ^{2} 2 \theta \\
& S_{B_{2 g}}=|f|^{2} \cos ^{2} 2 \theta
\end{aligned}
$$

where $\emptyset_{c a}$ is the phase difference $\emptyset_{c}-\emptyset_{a}$. As described, the Raman intensity of $A_{1 g}$ and $B_{2 g}$ modes shows different periodic variation with the sample rotation angle under parallel and cross-polarization configurations of the scattered light. According to (3) and (5), under the parallel configuration, when the crystal orientation parallel to the scattered light, the Raman intensity of $A_{1 g}$ mode has maximum or minimum value, while $B_{2 g}$ mode 
is forbidden. In contrast, under the cross-polarization configuration, the $A_{1 g}$ mode is forbidden, and Raman intensity of $B_{2 g}$ mode has the maximum value. 